\title{
POLYMER NANOCOMPOSITES: SYNTHETIC AND NATURAL FILLERS A REVIEW
}

William Gacitua E. ${ }^{1}-$ Aldo Ballerini A. ${ }^{2}-$ Jinwen Zhang $^{3}$

\begin{abstract}
This paper reviews current research, techniques for characterization and trends on the field of nanocomposites. Nanocomposites are new materials made with fillers which have nanosize. These materials have a big potential for applications in the automotive and aerospace industry as well as in construction, electrical applications and food packing. There is a tremendous interest for using bionanoparticles like cellulose microfibrils or whiskers to be applied in the new era of biocomposites.
\end{abstract}

Keywords: nanoparticles, natural nanofibers, biopolymers, composites

\section{INTRODUCTION}

The particles with small size in the range from a few to several tens of nanometers are called quasi zero-dimensionalmesoscopic system, quantum dots, quantized or Qparticles, etc. (Sharma, et. al, 2004). According Jordan et. al (2004) the nano-sized inclusions are defined as those that have at least one dimension in the range 1 to $100 \mathrm{~nm}$.

Nanotechnology is now recognized as one of the most promising areas for technological development in the $21^{\text {st }}$ century. In materials research, the development of polymer nanocomposites is rapidly emerging as a multidisciplinary research activity whose results could broaden the applications of polymers to the great benefit of many different industries. Polymer nanocomposites (PNC) are polymers (thermoplastics, thermosets or elastomers) that have been reinforced with small quantities (less than $5 \%$ by weight) of nano-sized particles having high aspect ratios $(\mathrm{L} / \mathrm{h}>300)$ (Denault and Labrecque, 2004). Figure $\mathrm{N}^{\circ} 1$ shows a classical layered silicate nanocomposites.

PNCs represent a radical alternative to conventional filled polymers or polymer blends - a staple of the modern plastics industry. In contrast to conventional composites, where the reinforcement is on the order of microns, PNCs are exemplified by discrete constituents on the order of a few nanometers. The value of PNC technology is not solely based on the mechanical enhancement of the neat resin nor the direct replacement of current filler or blend technology. Rather, its importance comes from providing value-added properties not present in the neat resin, without sacrificing the resin's inherent processibility and mechanical properties, or by adding excessive weight. PNCs contain substantially less filler (1-5 vol \%) and thus enabling greater retention of the inherent processibility and toughness of the neat resin (Vaia and Wagner, 2004).

\footnotetext{
Assistant professor, Depto. Ingenieria en Maderas. Universidad del Bío- Bío, Concepción-Chile. Investigador CIPA. wgacitua@mail.wsu.edu Associate professor, Depto. Ingenieria en Maderas. Universidad del Bío-Bío, Concepción-Chile. Investigador CIPA. aballeri@ubiobio.cl

${ }^{3}$ Assistant professor, Civil \& Environmental Engineering Department, Washington State University, Pullman WA USA. jwzhang@wsu.edu Corresponding author:wgacitua@mail.wsu.edu

Received: June 06, 2005. Accepted: October 09, 2005.
} 


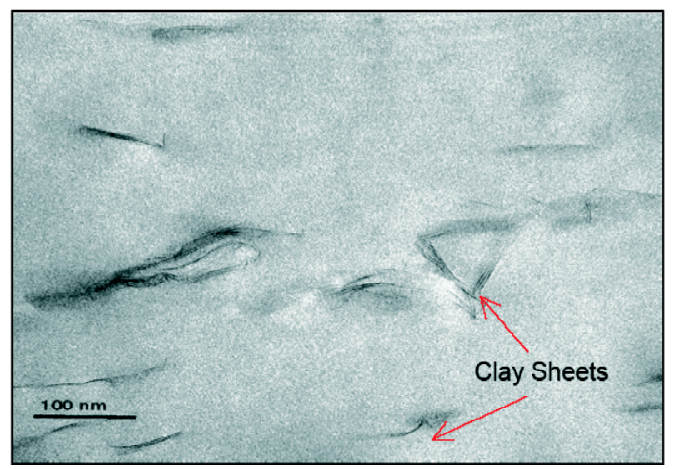

Figure $\mathbf{N}^{\circ} 1$ : Transmission electron microscopy (TEM) of a polymer/layered silicate nanocomposites prepared in a twin screw extruder (Denault and Labrecque, 2004).

This issue provides a snapshot of the rapidly developing PNC field and a summary of two of the most investigated nanoparticles - layered silicates and carbon nanotubes. According Vaia and Wagner (2004), development of PNCs, as with any multicomponent material, must simultaneously balance four interdependent areas: constituent selection, cost-effective processing, fabrication, and performance. For PNCs, a complete understanding of these areas and their interdependencies is still in its infancy, and ultimately many perspectives will develop, dictated by the final application of the specific PNC.

To convey the origin and interrelation of these distinguishing characteristics, Figure $\mathrm{N}^{\circ} 2$ compares the dominant morphological scale of a classic filled polymer containing $1 \mu \mathrm{m}$ × $25 \mu \mathrm{m}$ fibers in an amorphous matrix to that of a nano-filled system at the same volume fraction of filler, but containing $1 \mathrm{~nm} \times 25 \mathrm{~nm}$ fibers.

There are three main material constituents in any composite: the matrix, the reinforcement (fiber), and the so-called interfacial region. The interfacial region is responsible for communication between the matrix and filler and is conventionally ascribed properties different from the bulk matrix because of its proximity to the surface of the filler (Vaia and Wagner, 2004).

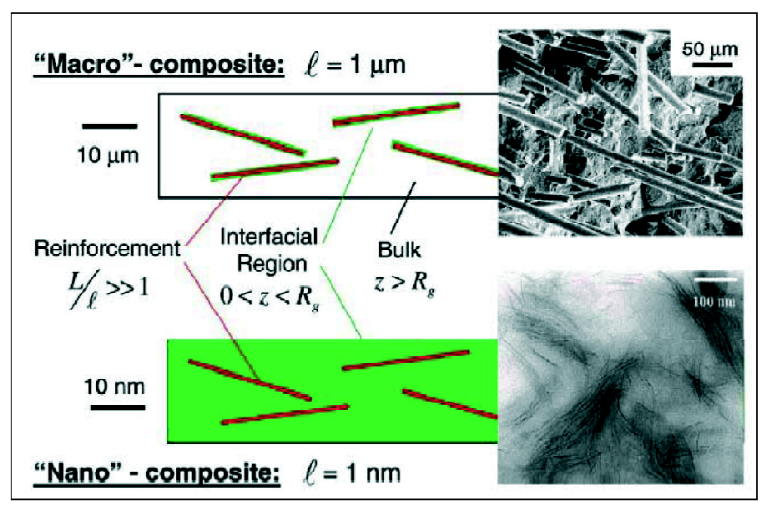

Figure $\mathbf{N}^{\circ}$ 2: Schematic comparison of a macro-composite containing $1 \mu \mathrm{m}$ x $25 \mu \mathrm{m}$ ( x L) fibers in an amorphous matrix to that of a nano-composite at the same volume fraction of filler, but containing $1 \mathrm{~nm} \times 25 \mathrm{~nm}$ fibers. Constituents in any composite: the matrix (white), the reinforcement (fiber, red), and the so-called interfacial region (green). Scanning electron micrograph shows E-glass reinforced polyolefin (15 $\mu \mathrm{m}$ fiber) and transmission electron micrograph shows montmorillonite-epoxy nanocomposite (1 nm thick layers) (Vaia and Wagner, 2004). 


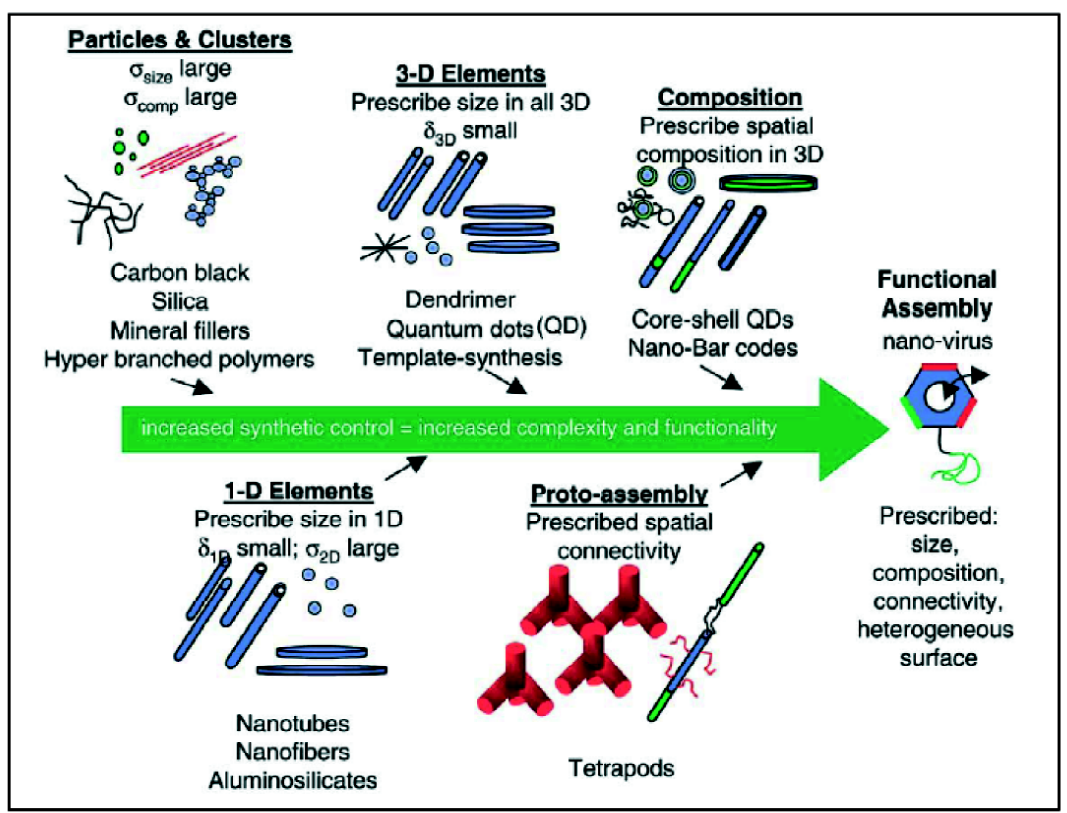

Figure $\mathbf{N}^{\circ}$ 3: Categorization of nanoparticles based on increasing functionality and thus, potential to increase functionality of the polymer matrix (Vaia and Wagner, 2004).

In almost every case, nanoparticles are added to the matrix or matrix precursors as $1-100 \mu \mathrm{m}$ powders, containing an association of nanoparticles. The overwhelming majority of the nanoparticles summarized in Figure $\mathrm{N}^{\circ} 3$ can be grouped into two categories based on this association: (i) lowdimensional crystallites and (ii) aggregates.

Layered silicates, single wall nanotubes (SWNTs), and other extreme aspect ratio, very thin (0.5-2 $\mathrm{nm}$ ) nanoparticles exhibit translational symmetry within the powder (Vaia and Wagner, 2004).

Polymer/layered nanocomposites in general, can be classified into three different types, namely (i) intercalated nanocomposites, (ii) flocculated nanocomposites, and (iii) exfoliated nanocomposites (see figure $\mathrm{N}^{\circ} 4$ ) (Wypych and Satyanarayana, 2005; Ray and Okamoto, 2003).

In the first case polymer chains are inserted into layered structures such as clays, which occurs in a crystallographically regular fashion, with a few nanometers repeat distance, irrespective of the ratio of polymer to layered structure. In the second case, flocculation of intercalated and stacked layers to some extent takes place due to the hydroxylated edge-edge interactions of the clay layers. Finally, separation of the individual layers in the polymer matrix occurs in the third type by average distances that depend only on the loading of layered material such as clay. In this new family of composite materials, high storage modulus, increased tensile and flexural properties, heat distortion temperature, decrease in gas permeability, and unique properties such as selfextinguishing behavior and tunable biodegradability are observed, compared to matrix material or conventional micro and macro-composite materials. Table $\mathrm{N}^{\circ} 1$ lists some examples of layered host crystals used in this type of composite.

There is a general agreement in the literature that exfoliated systems lead to better mechanical properties, particularly higher modulus, than intercalated nanocomposites (Jordan et. al, 2005). Figure $\mathrm{N}^{\circ} 5$ shows an ideal picture how polymers surface active agents favor in a subsequent separation of the platelets from each other forming finally the matrix material with homogeneously dispersed platelets (molecular composites) (Fischer, 2003). 
Table $\mathbf{N}^{\circ}$ 1: Example of layered host crystals susceptible to intercalation by a polymer (Wypych and Satyanarayana, 2005).

\begin{tabular}{|l|c|}
\hline Chemical nature & Examples \\
\hline Element & Graphite \\
\hline Metal chalcogenides & $(\mathrm{PbS})_{1.18}\left(\mathrm{TiS}_{2}\right)_{2}, \mathrm{MoS}_{2}$ \\
\hline Carbon oxides & Graphite oxide \\
\hline Metal phosphates & $\mathrm{Zr}\left(\mathrm{HPO}_{4}\right)_{2}$ \\
\hline Clays and layered silicates & $\begin{array}{l}\text { Montmorillonite, hectorite, saponite, fluoromica, } \\
\text { fluorohectorite, vermiculite, kaolinite, magadiite }\end{array}$ \\
\hline Layered double hydroxides & $\mathrm{Mg}_{6} \mathrm{Al}_{2}(\mathrm{OH})_{16} \mathrm{CO}_{3} n \mathrm{H}_{2} \mathrm{O} ; \mathrm{M}=\mathrm{Mg} . \mathrm{Zn}$ \\
\hline
\end{tabular}

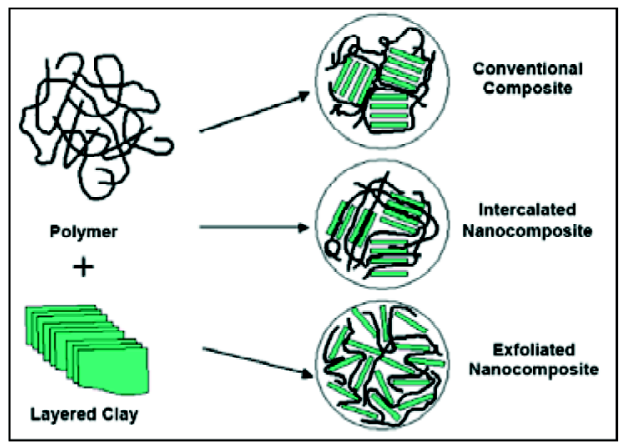

Figure $\mathbf{N}^{\circ}$ 4: Polymer-layered nanocomposites (Denault and Labrecque, 2004).

The exfoliation of layered minerals and hence the preparation of a homogeneous nanocomposite material is seriously hindered by the fact that sheet-like materials display a strong tendency to agglomerate due to their big contact surfaces. Figure $\mathrm{N}^{\circ} 6$ shows a graph of the surface area to volume ratio $\mathrm{A} / \mathrm{V}$ for a cylindrical particle with a given volume plotted vs. the aspect ratio $\alpha=1 / \mathrm{d}$ (Fischer, 2003).

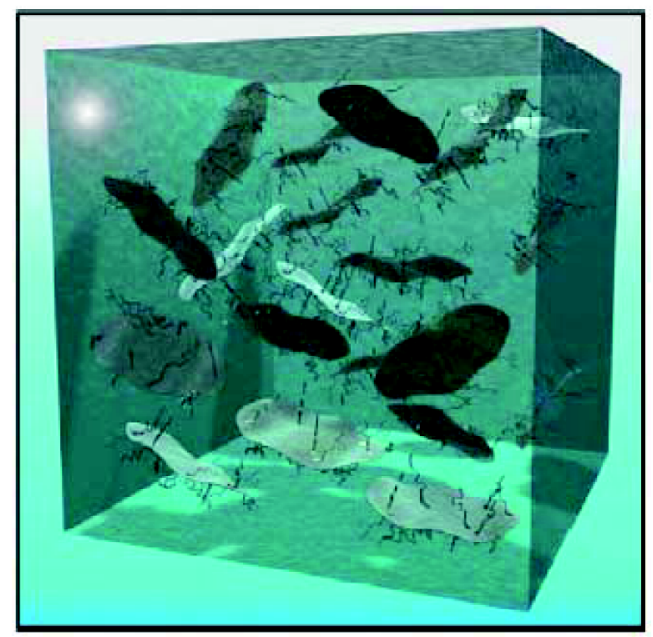

Figure $\mathbf{N}^{\circ}$ 5: Schematic picture of a polymer-clay nanocomposite material with completely exfoliated (molecular dispersed) clay sheets within the polymer matrix material (Fischer, 2003). 


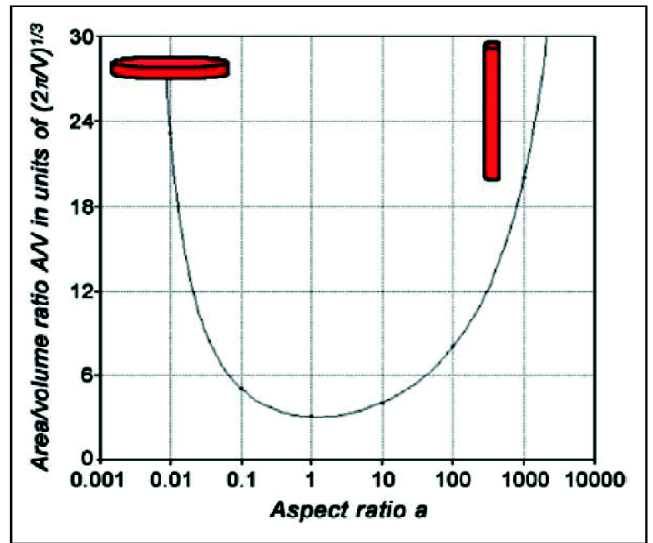

Figure $\mathbf{N}^{\circ}$ 6: Plot of the function describing the ratio of surface area to volume (A/V) vs. aspect ratio for cylindrical particles with a given volume. The $\mathrm{A} / \mathrm{V}$ value increases much quicker with respect to the aspect ratio for sheets compared to rods (Fischer, 2003).

The A/V values increase much steeper, with respect to the aspect ratios for sheets compared to rods. As a consequence, an incorporation of single surface modified inorganic fibres in a nanometer scale seems rather easy, since the contact surface between the fibres is rather small compared to those of sheet-like materials. Furthermore, the mechanical (reinforcing) potential of fibres is higher than that of sheets as recently described theoretically by Gusev (2001) and van Es (2001) (see Figure $\mathrm{N}^{\circ} 7$ ).

\section{NANOPARTICLES, METHOD OF PREPARATION}

Nanoparticles are obtained from available natural resources and generally they need to be treated because the physical mixture of a polymer and layered silicate may not form a nanocomposite; in this case a separation into discrete phases takes place. The poor physical interaction between the organic and the inorganic components leads to poor mechanical and thermal properties. In contrast, strong interactions between the polymer and the layered silicate nanocomposites lead to the organic and inorganic phases being dispersed at the nanometer level. As a result, nanocomposites exhibit unique higher properties than conventional composites (Biswas and Ray, 2001).

Solids with nanosize particle size cannot be prepared or treated by traditional methods simply because the reactants are not mixed on the atomic scale. All the alternative methods, e.g., hydrothermal, sol-gel, Pechini, chemical vapor deposition, and microwave, address this problem by achieving atomic scale mixing of reactants, in gas, liquid, or even solid phases. Most of these are low temperature methods, although finally firing may be required at high temperatures especially for ceramic-type products. These methods enable the final product with the following characteristics (Sharma et. al, 2004): Nanosize particles - Narrow particle size distribution - High surface area- Homogenous - Pure - Improved properties. 


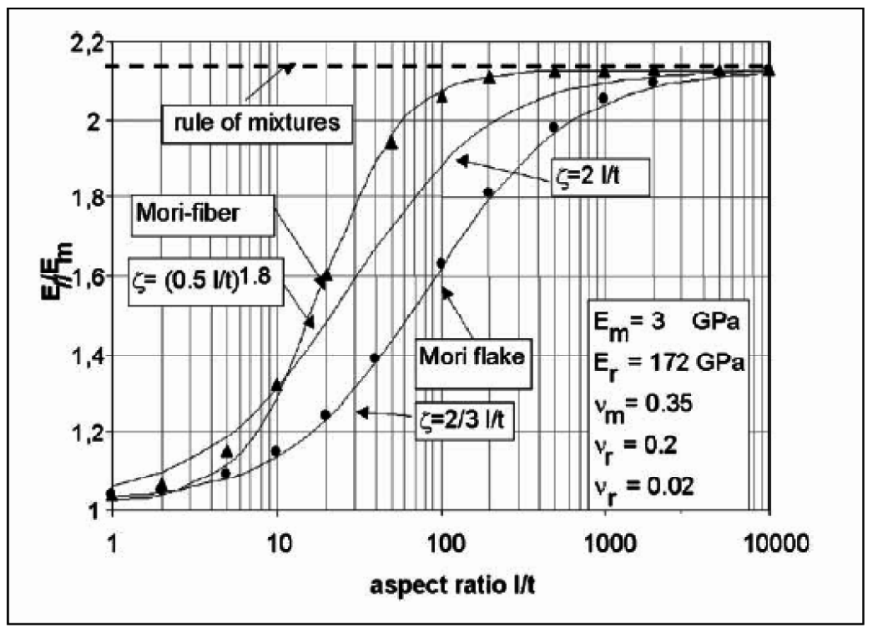

Figure $\mathbf{N}^{\circ}$ 7: Plot of the computed Young's modulus of a unidirectional composite filled with platelets or fibres of different aspect ratios using the Tsai- Halpin and the Mori-Tanaka model. In both cases, a stronger reinforcing action of the fibres compared to platelets can be predicted

(van Es 2001).

The methods are (Sharma et. al, 2004):

Hydrothermal Synthesis

Hydrothermal reactions are usually performed in closed vessels. The reactants are either dissolved or suspended in a known amount of water and are transferred to acid digestion reactors or autoclaves. Under hydrothermal conditions, reactants otherwise difficult to dissolve can go into solution and reprecipitate.

\section{$\underline{\text { Sol-Gel Synthesis }}$}

Sol-gel synthesis is a very viable alternative method to produce nanocrystalline elemental, alloy, and composite powders in an efficient and cost-effective manner. Nanocrystalline powders could be consolidated at much lower pressures and temperatures.

\section{Polymerized Complex Method}

Wet chemical method using polymeric precursor based on the Pechini process has been employed to prepare a wide variety of ceramics oxides. The process offers several advantages for processing ceramic powders such as direct and precise control of stoichiometry, uniform mixing of multicomponents on a molecular scale, and homogeneity.

\section{Chemical Vapor Deposition}

Chemical vapor deposition (CVD) may be defined as the deposition of a solid on a heated surface from a chemical reaction in the vapor phase. It is a versatile process suitable for the manufacturing of coatings, powders, fibers, and monolithic components.

\section{Microwave Synthesis}

Recently, there has been a growing interest in heating and sintering of ceramics by microwaves. The field of application in the use of microwave processing spans a number of fields from food processing to medical applications to chemical applications. Major areas of research in microwave processing for ceramics includes microwave material interaction, dielectric characterisation, microwave equipment design, new material development, sintering, joining, and modeling. A microwave chemical deposition 
unit is used for the fabrication of carbon nanotubes and coils. It consists of microwave magnetron, circulator, four-stub tuner, waveguide, cavity, etc.

\section{High-Energy Ball Milling Processes}

Ball milling has been utilized in various industries to perform size reduction for a long time. Recently, materials with novel microstructures and properties have been synthesized successfully via high-energy ball milling processes. Although different terms have been used to describe the high-energy ball milling processes, three terms are generally used to distinguish powder particle behavior during milling: mechanical alloying (MA), mechanical milling (MM), and mechanochemical synthesis (MS). There are some inherent advantages in processing nanomaterials via high-energy ball milling techniques, such as excellent versatility, scalability, and cost-effectiveness. Therefore high-energy ball milling techniques are well suited for manufacturing large quantity of nanomaterials.

\section{FORMULATIONS AND FUNCTIONS}

Ellis and D'Angelo (2003) prepared and characterized experimental polypropylene (PP) nanocomposites, containing approximately $4 \mathrm{wt} \%$ of an organophilic montmorillonite clay, and their properties were compared with those of talc- filled (20-40 wt \%) compositions. They found that it is possible to reduce weight maintaining or even improved flexural and tensile modulus, especially at temperatures up to $70^{\circ} \mathrm{C}$. Also, TEM micrographs shown in Figure $\mathrm{N}^{\circ} 8$ also support the inference of an intercalated PP nanocomposites rather than a fully exfoliated nanocomposite. The micrographs indicate a well-dispersed morphology with incomplete exfoliation.

The classical view of fiber reinforced composites implies that strong fiber-matrix interfaces lead to high composite stiffness and strength, but also to low composite toughness because of the brittleness of the fiber and the absence of crack deflection at the interface. Vice versa, composites with weak interfaces usually have relatively low stiffness and strength, but higher toughness. One of the most difficult problems in the physics of polymer nanocomposites is the measurement of the extent and efficiency of stress transfer through the interface between nanoparticles and matrix.

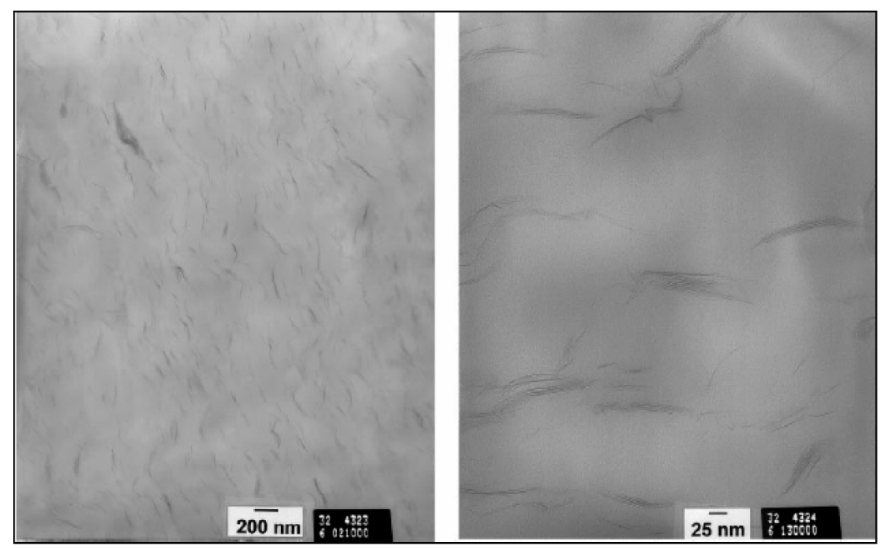

Figure $\mathbf{N}^{\circ} \mathbf{8}$ : TEM micrographs of the PP nanocomposites (Ellis and D’Angelo, 2003). 
It is a general knowledge that the larger is their internal surface and hence their tendency to agglomerate rather than to disperse homogeneously in a matrix. Also, the contact surface in such dispersion between the elements and the matrix material grows dramatically and consequently the problems in creating an intense interaction at this interface (Fischer, 2003). Fischer reported that an agglomeration of the clay platelets in the organic- inorganic hybrid coatings did not occur up to an amount of $20 \mathrm{wt} . \%$ based on the solid content of the coating material; the nanocomposite coatings of both organic and organic- inorganic hybrids remained transparent up to an amount of $15 \mathrm{wt} . \%$ of clay. A homogeneous dispersion of the clay platelets (5.0 wt.\% based on solid coating material) in an organicinorganic hybrid coating was observed using TEM.

Current micromechanics theories rely on the idea that the effective properties of composite materials, such as Young's modulus, are functions of properties of constituents, volume fraction of components, shape and arrangement of inclusions, and matrix-inclusion interface. These theories, therefore, predict that the properties of composite materials are independent of the size of inclusions. In general, this is correct for systems with micron size reinforcement, but, as mentioned above may not be true for nanocomposite systems (Jordan et. al, 2005).

The effects of the nanoparticles are dependent on many variables but especially upon the relative crystalline or amorphous nature of the polymer matrix as well as the interaction between the filler and matrix (Jordan et. al, 2005). Jordan et al. state that trends are observed but no universal patterns for the behavior of polymer nanocomposites can be deduced in general.

\section{TECHNIQUES FOR CHARACTERIZATION}

Experimental techniques used for the characterization of nanocomposites include NMR for materials behavior (giving greater insight into the morphology, surface chemistry, and to a very limited extent the quantification of the level of exfoliation in polymer nanocomposites), X-ray diffraction (due to ease and availability), transmission electronmicroscopy (TEM-allows a qualitative understanding of the internal structure, spatial distribution of the various phases, and direct visualization of defect structure), differential scanning calorimetry (DSC - to understand the nature of crystallization taking place in the matrix), FTIR (to detect functional groups and understand the structure of the nanocomposites), dynamic mechanical analysis (DMA - response of a material to oscillatory deformation as a function of temperature, giving storage modulus [corresponds to elastic response to deformation], loss modulus [corresponds to plastic response to deformation], and $\tan \delta$ [ratio of the previous two and an indicator of occurrence of molecular mobility transitions]), and resonance Raman spectroscopy (for structural studies) (Ellis and D'Angelo, 2003; Wypych and Satyanarayana, 2005; Ray and Okamoto, 2003).

The atomic force microscope (AFM) is another equipment to characterize nanocomposites (Greene et al. 2004). AFM can provide information about the mechanical properties of a surface at a length scale that is limited only by the dimensions of the AFM tip. From commercial suppliers, AFM tips with $10-\mathrm{nm}$ radius of curvature are readily available. When probing mechanical properties, the attractive and repulsive force interactions between the tip and sample are monitored.

In the future research, some interesting studies will be conducted (Denault and Labrecque, 2004; Ellis and, D'Angelo, 2003; Wypych and Satyanarayana, 2005; Ray and Okamoto, 2003): Processing of Polymer Nanocomposites (Injection and micro-injection moulding, Foam extrusion, Blow moulding, Film blowing, Glass or carbon fibre reinforced nanocomposites).

Polymer Nanocomposites Behaviour and Performance (viscous and viscoelastic effects, Thermal heat capacity, phase transitions, crystallization kinetics, fire resistance, degradation, Thermodynamic, 
Short and long term mechanical and physico-chemical performance, permeability, stress deformation, fracture behaviour, impact, fatigue, environmental resistance, Microstructure development during processing (orientation, crystallinity, residual stresses).

Nanoparticles Surface Modification Routes for Specific Applications (Nanoparticles [layered clays, carbon nano-tubes (CNT) or others] surface modification via chemical routes, Compatibilization techniques for optimum interaction between polymer matrix and Nanoparticles).

\section{Development of Melt Blending Processes}

(Optimization of twin screw extruders (screw configuration and processing conditions) for optimal mixing/dispersion of Nanoparticles, Optimization of mixing/dispersion with motionless or dynamic extensional flow mixers, Development of coupled flow/heat-transfer/mixing models).

\section{PROCESSING CONDITIONS}

The traditional routes to prepare nanocomposites using layered compounds as reinforcement, especially clays, can be summarized as follows (Wypych and Satyanarayana, 2005; Ray and Okamoto, 2003, Ku et al, 2004):

\section{Exfoliation/adsorption}

First the layered host is exfoliated in a solvent, in which the polymer is soluble (water, toluene, etc). The polymer is adsorbed onto the single-layer surfaces and after evaporation of the solvent or a precipitation procedure, the single layers are restacked, trapping the polymer and the hydrated/ solvated ionic species (see figure $\mathrm{N}^{\circ}$ ).

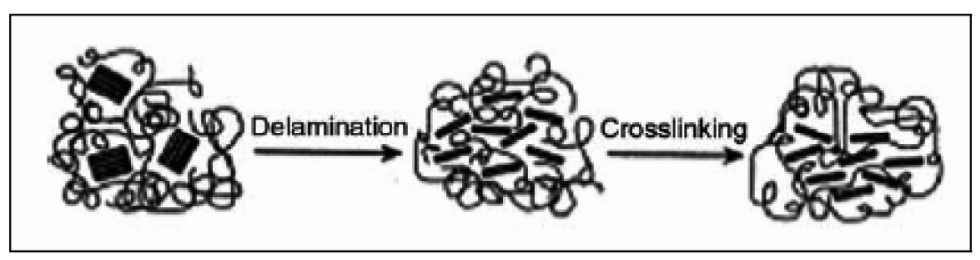

Figure $\mathbf{N}^{\circ}$ 9: Schematic illustration of nanocomposite synthesis (Ray and Okamoto, 2003).

In situ intercalative polymerization

Polymer is formed (initiation of polymerization by heating or radiation or by diffusion) between the layers by swelling the layer hosts within the liquid monomer or monomer solution (see figure $\mathrm{N}^{\mathrm{o}} 10$ ).

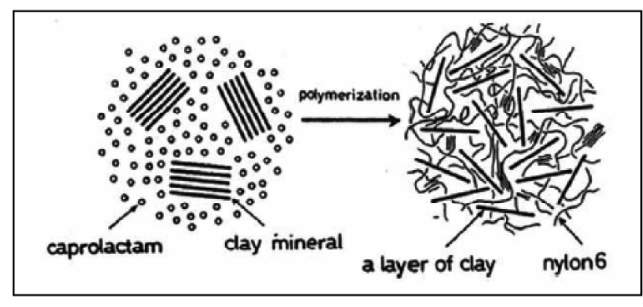

Figure $\mathbf{N}^{\circ} \mathbf{1 0}$ : Schematic illustration for synthesis of Nylon-6/clay nanocomposites (Ray and Okamoto, 2003). 


\section{Melt intercalation}

This method, an environmentally benign one, uses all types of polymers as well as being compatible with practicing polymer industrial processes such as injection molding, being the most popular procedure to prepare nanocomposites for industrial applications. In this method, polymers, and layered hosts are annealed above the softening point of the polymer (see figure $\mathrm{N}^{\circ} 11$ ).

\section{Template synthesis}

In situ layered double hydroxide (LDH) based nanocomposites can be obtained in a template of polymer aqueous solution for the formation of host layers and usually employed for water-soluble polymers.

\section{Intercalation of prepolymer from solution}

The layered host is to be swelled in a solvent (water, toluene, etc.) followed by its mixture with polymer or prepolymer, whereby the chains of the latter intercalate while displacing the solvents used for swelling. Polymer layered nanocomposite results when the solvent within the interlayer is removed.

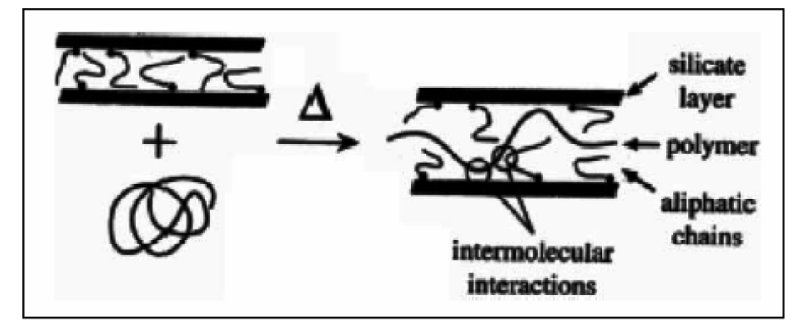

Figure $\mathbf{N}^{\circ} 11$ : Schematic depicting the intercalation process between a polymer melt and an organic modified layered silicate (Ray and Okamoto, 2003).

\section{RESULTS AND APPLICATIONS}

\section{Current research}

Jordan et al. (2005) reported result of composites with polypropylene matrix and calcium carbonate $\left(\mathrm{CaCO}_{3}\right)$ nanoparticles. In their system the $\mathrm{CaCO}_{3}$ inclusions had an average size of $44 \mathrm{~nm}$ and a strong interaction with the polymer matrix. The addition of $\mathrm{CaCO}_{3}$ nanoparticles to a PP matrix produced an increase in the elastic modulus compared to the pure matrix. The increase in modulus coincided with an increase in nanoparticle volume fraction.

Clay-reinforced nanocomposites have received considerable attention in recent years (more than 100 articles have been published in the literature on clay composites in the past three years). A number of polymers, such as PC, PAN, PP, etc. were used as the matrix. Shelley et al. (2001) examined a polyamide- 6 system with clay platelets. The platelets constituted $2 \%$ and $5 \%$ weight fraction and were $1 \mathrm{~nm} \times 10 \mathrm{~nm} \times 10 \mathrm{~nm}$ in size. Good interaction was found between the matrix and inclusions. With this setup, the elastic modulus was found to improve for both the $2 \%$ and $5 \%$ samples. For the smaller weight fraction (2\%), the increase in effective elastic modulus was $40 \%$ over the modulus of the pure polymer system. The larger weight fraction (5\%) improved the effective modulus by a factor of two as compared to that of pure polymer. These results were for tensile specimens cut in both longitudinal and transverse directions. In addition, the yield stress also improved for both weight fractions, with the greatest improvement found for the higher concentration of inclusions. The other property studied was the strain-to-failure. The $2 \%$ system was found to give higher strain-to-failure than the pure system in 
the longitudinal direction but close to that in the pure system in the transverse direction. The higher filler content resulted in a decline in strain-to-failure from the pure system in both directions.

From the above discussion, it is possible to extract a few trends for the behavior of polymer matrix nanocomposites based on the nature of the polymer matrix, particularly crystalline or amorphous nature of the polymer, and the interaction between the filler and matrix. The elastic modulus tends to increase with the volume fraction of inclusions in every case. In some systems, there is a critical volume fraction at which aggregation occurs and the modulus goes down. In general, there is also an increase in modulus as the size of the particle decreases. Interaction between matrix and filler may play an important role in the effects of the nanoparticles on composite properties. The overall trend of the modulus of polymer nanocomposites is not found to be greatly dependent upon the nature of the matrix nor the interaction between filler and matrix. An examination of the yield stress gives a different trend than that of the elastic modulus. For composites with good interaction between filler and matrix, the yield stress tends to increase with increasing volume fraction and decreasing particle size, similarly to the increase in modulus under same conditions. The pattern changes when there is poor interaction between the matrix and particles. The addition of nanoparticles with poor interaction with the matrix causes the yield stress to decrease, compared to the neat matrix, regardless of the filler concentration or size. The ultimate stress follows a similar pattern as that observed for the yield stress. It generally increases in polymer systems (both crystalline and amorphous) with good filler-matrix interaction and it increases in general as particle size decreases. There is no uniform trend with respect to the volume fraction of particles for the ultimate stress. A poor filler-matrix interaction leads to a decrease in the ultimate and yield stress as compared to the pure matrix system (Jordan et. al, 2005). Jordan et. al (2005) state that in general, viscoelastic properties tend to be higher in nanocomposites than in pure polymer systems. When there is good filler-matrix interaction, the storage modulus generally increases with increasing volume fraction. The modulus also seems to increase as the particle size decreases. However, there is little experimental work in this area for composites with poor filler-matrix interactions. Overall, the storage modulus tends to increase with the presence of nanoparticles in a composite system. Morphological details, such as exfoliation, intercalation, or cross-linked matrix versus uncross-linked matrix, have a significant effect on the viscoelastic properties of nanocomposites.

The crystallinity of crystalline and semi-crystalline polymers is not affected very much by the addition of nanoparticles. There may be some changes in particular nanocomposite systems, but overall no major differences in crystallinity of nanocomposites versus neat polymers were observed in any of the systems examined. On the other hand, the glass transition temperature was influenced by the addition of particles. When there is good filler-particle interaction, the glass transition temperature tends to increase with a decrease in the size of particles for amorphous polymers. For crystalline polymers, the transition temperature decreases with an increase in particle concentration. For an amorphous system with poor filler-polymer interfacial interaction, the glass transition temperature decreased overall. Thus, while the degree of crystallinity is not significantly affected by the presence of particles, the glass transition temperature is very dependent upon this factor (Jordan et. al, 2005).

Chan et al. (2002) proposed that properties such as elastic modulus, tensile strength, and yield strength decrease in nanocomposites with polypropylene matrix due to the change in nucleation caused by the nanoparticles (Figure $\mathrm{N}^{\circ} 12$ ). The nanoparticles produce a much larger number of nucleating sites but, in turn, greatly reduce the size of these spherulites. In their experimental work, no spherulites were found in the nanocomposites by SEM indicating that either none were present or they were reduced to such a small size that SEM could not detect them. It was further proposed that there was another mechanism which was causing these same properties to increase. The increase occurred when there was a strong interaction between the polymer and filler. This interaction had larger impact in nanocomposites due to the large interfacial area between the filler particles and the matrix. Jordan et al. (2005) stated that for most systems, density is proportional to elastic modulus, so the region directly 
surrounding the inclusions will be a region of high modulus. If the particles are densely packed, then the boundary layer of polymer at the interface will comprise a large percentage of the matrix and can create a system where there is no space for a low modulus region to form.

The small interparticle distance in nanocomposites was used as another parameter to explain the changes in the elastic modulus and strength of these materials when compared with the composites with micron-sized particles. The same parameter also plays a role in the glass transition temperature changes observed in nanocomposites versus composites with micron-sized reinforcement (Jordan et. al, 2005). When there is little or no interfacial interaction between the filler and matrix and the interparticle distance is small enough, the polymer between two particles acts as a thin film. For a thin film, the glass transition temperature decreases as film thickness decreases. The distance between particles in a composite with the filler weight fraction below $0.5 \%$ is relatively large, and hence, in this case the polymer between each particle is not considered to belong to the thin film regime. As the filler concentration increases, the interparticle distance and the resulting thickness of the film, decrease. Finally, Jordan et al. established that for constant filler content, with reduction in particle size, number of filler particles increases, bringing the particles closer to one another. Thus, the interface layers from adjacent particles overlap, altering the bulk properties significantly.

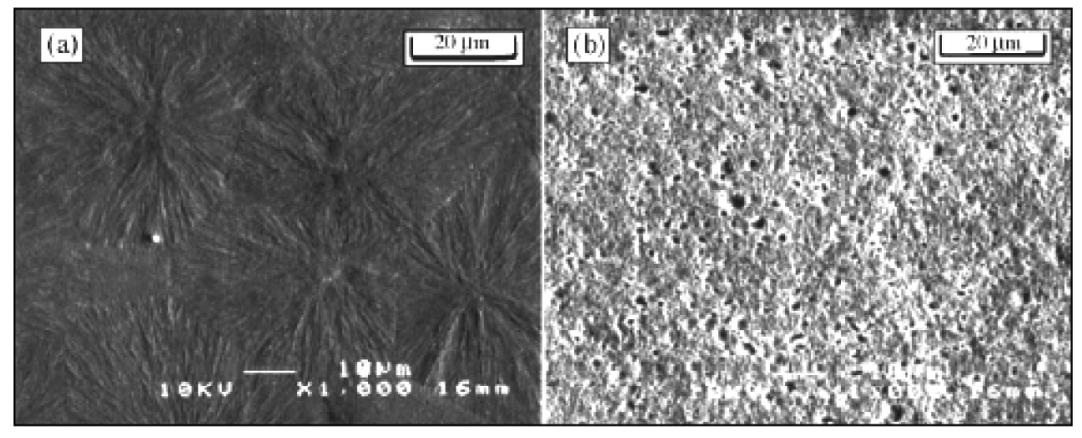

Figure $\mathbf{N}^{\circ} 12$ : (a) Pure polypropylene and (b) polypropylene with $9.2 \%$ volume filler (Chan et al. 2002).

On the other hand, Ash et al. (2004) stated that the most dramatic increases in the modulus of nanocomposites occur in the region above the $\mathrm{Tg}$ (glass transition termperature). Often, these increases are much greater $(4000 \%)$ than those that occur below Tg. This is hypothesized to be due to the creation of crosslinks, either temporary or permanent, between nanoparticles and polymer which serve to increase the plateau modulus. Indeed, the ability to strictly control the size and surface activity of nanoparticles in recent dynamic mechanical studies on silica/polyvinylacetate nanocomposites above the $\mathrm{Tg}$ has shed new light on the nonlinear reinforcement behavior of rubbery melts.

Ray and Okamoto (2003) presented a brief discussion about heat distortion temperature (HDT) of nanocomposites. HDT of a polymeric material is an index of heat resistance towards applied load. Most of the nanocomposite studies report HDT as a function of clay content, characterized by the procedure given in ASTM D-648 (see figure $\mathrm{N}^{\circ} 13$ ). Ray and Okamoto state that increasing of HDT due to clay dispersion is a very important property improvement for any polymeric material, not only from application or industrial point of view, but also because it is very difficult to achieve similar HDT enhancements by chemical modification or reinforcement by conventional filler. 


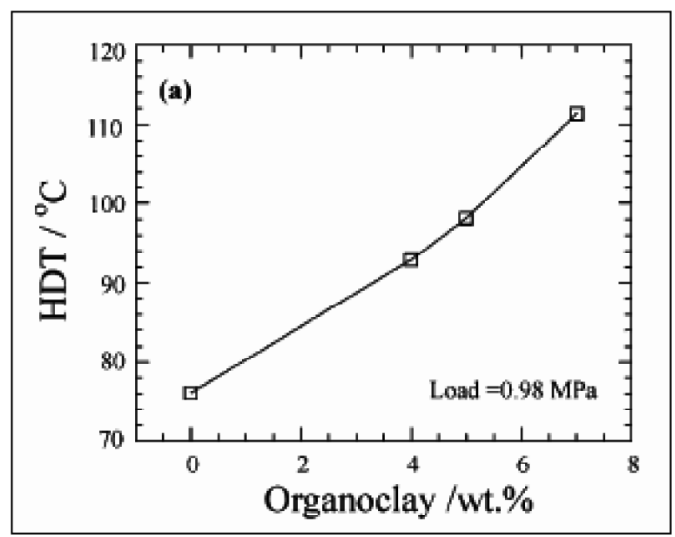

Figure $\mathbf{N}^{\circ}$ 13: Organoclay (wt\%) dependence of HDT (Ray and Okamoto, 2003).

Tidjani (2005), found a dramatic reduction in peak heat release rate for the nanocomposites compared to pure polypropylene-graft-maleic anhydride and its hybrid. He states that the improved flammability happens in the condensed phase and is not likely to be due to a higher thermal stability of the nanocomposite. The impermeability of the silicate layers in the polymer, which reduced the diffusion of gases in the nanocomposites, may participate in the reduction of the flammability.

Using an intercalated thermoplastic polyolefin (TPO)/organoclay nanocomposite with maleic anhydride functionalized PP as a compatibilizer, Mishra et al. (2005), established that the compatibilizer not only enhances the intercalation of the polymer chain inside the clay gallery but also changes the thermoplastic elastomer composition (which is very important for end use application) of the TPO/ clay nanocomposite. The tensile modulus as well as storage modulus of TPO/organoclay nanocomposite was substantially higher over a $20 \%$ talc based microcomposite.

From the point of view of gas barrier properties, nanocomposites offer interesting features. Ray and Okamoto (2003) proposed that clays increase the barrier properties by creating a maze or "tortuous path" (see Figure $\mathrm{N}^{\mathrm{o}} 14$ ) that retards the progress of the gas molecules through the matrix resin.

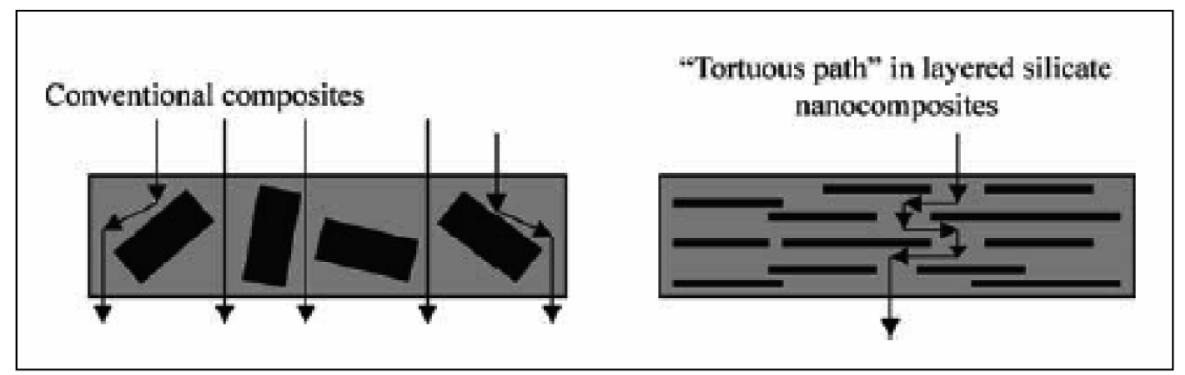

Figure $\mathbf{N}^{\circ} 14$ : Formation of tortuous path in polymer/layered silicate nanocomposites (Ray and Okamoto, 2003).

In the field of nanocomposites using carbon nanotubes (CNTs), it has already been established that CNTs possess remarkable properties. Nowadays, the main challenge is to be able to implement these properties in composites on a macroscale, combining the choice of materials with the appropriate processing method. Table $\mathrm{N}^{\circ} 2$ shows a comparison between CNTs composite and polypropylene matrix (Breuer and Sundarraraj, 2004). 
Table $\mathbf{N}^{\circ}$ 2: Properties of Fibers From Polypropylene (PP) and PP/CNT Composites (Breuer and Sundarraraj, 2004).

\begin{tabular}{|lcccc|}
\hline \multicolumn{1}{c}{ Sample } & $\begin{array}{c}\text { Tensile Strength } \\
\text { (MPa) }\end{array}$ & $\begin{array}{c}\text { Tensile Modulus } \\
\text { (GPa) }\end{array}$ & $\begin{array}{c}\text { Elongation to } \\
\text { Break (\%) }\end{array}$ & $\begin{array}{c}\text { Compressive } \\
\text { Strength (MPa) }\end{array}$ \\
\hline PP-control & $490 \pm 60$ & $4.6 \pm 0.7$ & $23 \pm 5$ & $25 \pm 1$ \\
PP +5 wt\% nano carbon fiber & $570 \pm 70$ & $7.1 \pm 0.9$ & $16 \pm 2$ & $48 \pm 10$ \\
\hline
\end{tabular}

Studies about photodegradation of nanocomposites found that Photo-oxidation at long wavelengths of polypropylene-based nanocomposites produces the same photoproducts as those of the pristine polypropylene and in the same quantities. The maleic anhydride grafted PP used as compatibilizer and the organically modified nanoclay do not significantly modify the rate of photooxidation of the samples. However, the efficiency of additives is considerably reduced. This could result from the location of the additives close to the organoclay due to the spreading of these polar species onto the hydrophilic nanoplatelets (Mailhota et al. 2003).

\section{Applications}

In the field of coating systems, Fischer (2003) reported that permeability of the nanocomposites coatings for water vapour markedly decreased with respect to the non-modified coating; a decrease of the water vapour permeability by a factor of 15 has been measured. This points to the presence of a strong bonding of the methylene blue to the clay platelets. Clay particles are homogeneously dispersed in the coating matrix, thus resulting in a fully transparent coating.

Inorganic-organic composites based on organoalkoxysilanes and other alkoxides have demonstrated their usefulness for hard coatings on eye-glass lenses. It has been shown that the addition of nanoparticles, especially in combination with epoxy silanes, which act as an inorganic as well as an organic crosslinking agent, leads to a substantial increase of the abrasion resistance of such systems without losing any transparency. Also, nanocomposites have been developed for the fabrication of low surface free energy coatings. With nanoparticles incorporated into the matrix, high abrasion resistance can be obtained. To promote good adhesion to different substrates, like metals, ceramics and plastics, adhesion promoters have been added (Schmidt, 2001).

Mechanical properties of CNTs suggest that they may be used as reinforcing fibers in high-toughness nanocomposites, where stiffness, strength and low weight are important considerations. There are numerous possible applications; some examples are aerospace structural panels, sporting goods, ultralightweight thin-walled space structures for use in space, and high stiffness-to-weight space mirror substrates. Applications relating to nonlinear optics include protection of optical sensors from highintensity laser beams. Additional applications involving the optical and electronic properties are electronemitting flat-panel displays, electromechanical actuators, light-emitting diodes; supercapacitors, field-effect transistors, subpicosecond optical switches and optical limiters (Breuer and Sundarraraj, 2004). Conducting polymer structures can be constructed at low loadings of nanotube fillers.

Nanocomposites offer improvements over conventional composites in mechanical, thermal, electrical and barrier properties. Furthermore, they can reduce flammability significantly and maintain the transparency of the polymer matrix. In the case of layered silicate (clay) nanocomposites, loading levels of 2 to $5 \%$ by weight result in mechanical properties similar to those found in conventional composites with 30 to $40 \%$ reinforcing material (Denault and Labrecque, 2004). These attractive 
characteristics already suggest a variety of possible industrial applications for polymer nanocomposites (Wypych and Satyanarayana, 2005):

- automotive (gas tanks, bumpers, interior and exterior panels)

- construction (building sections and structural panels)

- aerospace (flame retardant panels and high performance components)

- electrical and electronics (electrical components and printed circuit boards)

- food packaging (containers and wrapping films)

According Silberglitt (2004), there are two possible paths or trends - a high-growth path under which nanocomposites materials are pervasively applied throughout society and a low-growth path under which the use of nanocomposites leads to incremental improvements in specific technology areas (see figure $\mathrm{N}^{\circ} 15$ ).

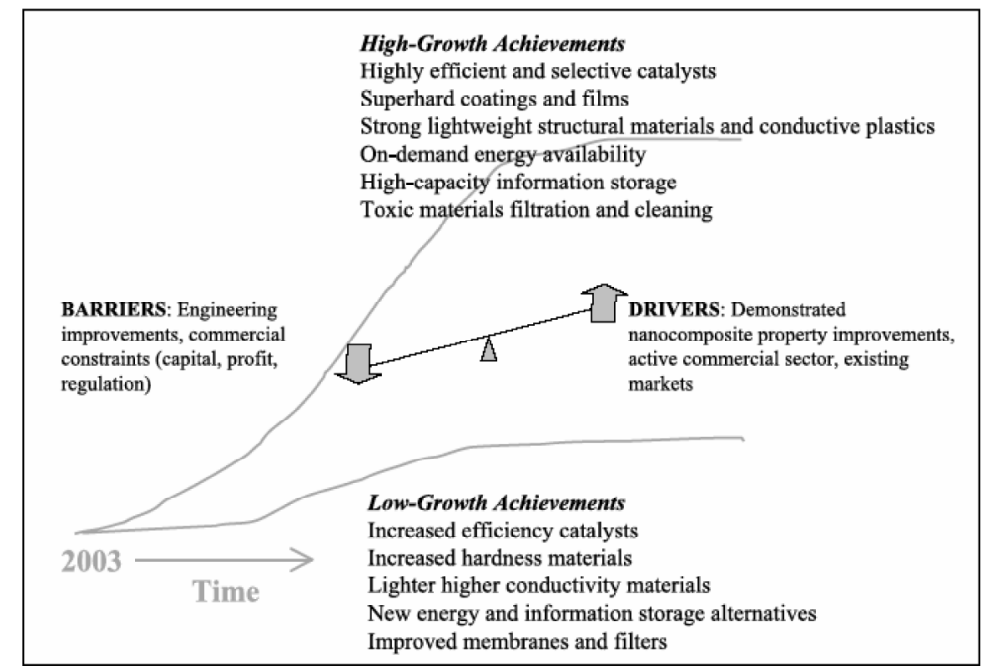

Figure $\mathbf{N}^{\circ}$ 15: Possible growth paths for nanocomposites applications (Silberglitt, 2004).

\section{CELLULOSE NANOCOMPOSITES; SPECIFIC APPLICATION}

There is an important source of nanoparticles and nanofibers in wood; Cellulose chains aggregate to form microfibrils, long threadlike bundles of molecules stabilized laterally by hydrogen bonds between hydroxyl groups and oxygens of adjacent molecules (see figure $\mathrm{N}^{\circ} 16$ ). The molecular arrangement of these microfibrillar bundles is sufficiently regular that cellulose exhibits a crystalline X-ray diffraction pattern. Depending on their origin, the microfibril diameters range from -2 to $20 \mathrm{~nm}$ for lengths that can reach several tens of microns. These microfibrils consist of monocrystalline cellulose domains with the microfibril axis parallel to the cellulose chains. There is also an appreciable amount of cellulose that is in an amorphous state within the microfibril (Helbert et al., 1996). Microfibril can be considered a string of polymer whiskers, linked along the microfibril by amorphous domains, and having a modulus close to that of the perfect crystal of native cellulose (estimated to $150 \mathrm{GPa}$ ) and a strength that should be - $10 \mathrm{GPa}$. The amorphous regions act as structural defects and are responsible for the transverse cleavage of the microfibrils into short microcrystals under acid hydrolysis. 
Cellulose microcrystals with dimensions of $5 \mathrm{~nm} \times 150-300 \mathrm{~nm}$ were obtained from wheat straw to evaluate the reinforcing effect of these fillers within a thermoplastic matrix, composites with a weight fraction of cellulose ranging from 0 to $30 \mathrm{wt} \%$ were processed by freeze-drying and molding a mixture of aqueous suspensions of microcrystals and poly(styrene-co-butyl acrylate) latex (Helbert et al., 1996). In this research, the mechanical properties are substantially improved by increasing the amount of filler. Due to the long preparation time for whiskers, microcrystalline wheat straw cellulose (or cellulose from other species) is a costly filler, despite the abundance and low price of the raw material. However, it can be useful for the processing of stiff small-size wares.

In the same way, Simonsen and Palaniyandi (2004) had started a project to study the use of cellulose nanocrystals to reinforce HDPE, mainly because cellulose nanocrystals offer exceptional strength, stiffness, low density, and they are derived from renewable resources and should be inexpensive. In this research, cellulose nanocrystals were prepared by acid hydrolysis of cotton. TEM analysis showed that the rod-shaped cellulose nanocrystals had an average length of $60-80 \mathrm{~nm}$ and width of 3 to $5 \mathrm{~nm}$; thus the aspect ratio was about 10 to 30 . The use of compatibilizers, or coupling agents, was investigated as a means of improving the dispersion of the nanocrystals and thus the mechanical properties of the composites. One compatibilizer was shown to improve the strength of the resulting composite.

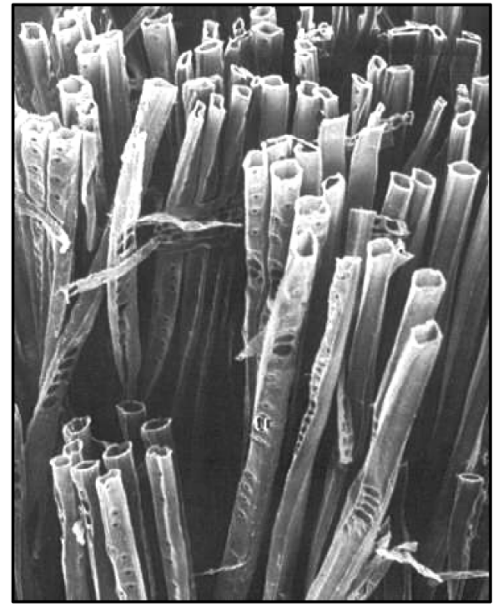

A

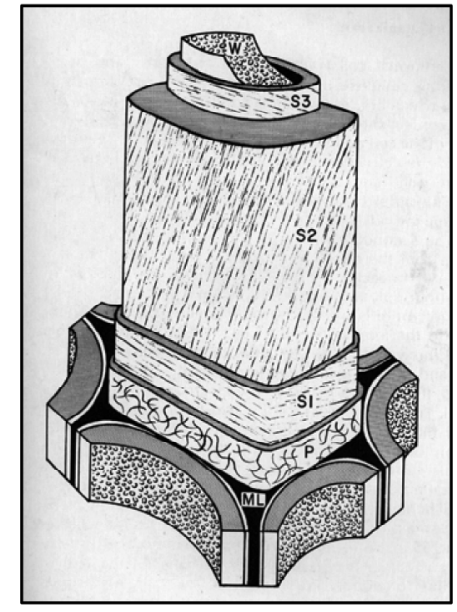

B

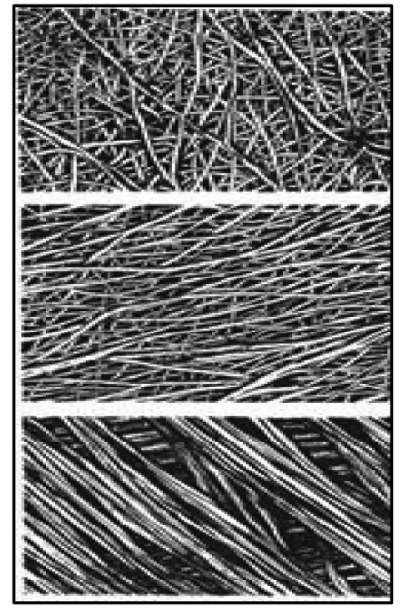

C

Figure $\mathbf{N}^{\circ}$ 16: Arrangement of microfibrils (nanofibers) in the cell wall.

A) Wood fiber or cell, B) Wood cell schematic C) Microfibrils (Winter, 2004).

Another group of researching is working with nanocrystals of cellulose; in his research Chatterjee (2004) suggests cellulose nanocrystals as a renewable, bio-based, low-density, reinforcing filler for polymer-based nanocomposites. They are trying to separate nanocrystas from surrounding amorphous regions by carefully controlled acid hydrolysis (amorphous regions are degraded more rapidly), see figure $\mathrm{N}^{\circ} 17$. Also, Grunert and Winter (2002) found that cellulose crystals exhibited reinforcement characteristics. They used nanocrystals obtained from fibers by acid hydrolysis of cellulose microfibrils. With elastic modulus of $138 \mathrm{GPa}$ for the crystalline phase and a calculated limiting specific surface area of several hundred $\mathrm{m}^{2} / \mathrm{g}$, cellulose nanocrystals have the potential for significant reinforcement of thermoplastic polymers at very small filler loadings. One restriction on the use of cellulose crystals as reinforcement is their incompatibility with a typically more hydrophobic thermoplastic matrix. To overcome this problem, cellulose nanocrystals from bacterial cellulose were topochemically 
trimethylsilylated. Both native and surface trimethylsilylated nanocrystals were employed as the particulate phase in nanocomposites with a cellulose acetate butyrate matrix (Grunert and Winter,2002). Matsumura et al. (2000) obtained a semitransparent composite sheets formed a thermoplastic cellulose hexanoate phase consolidated into a continuous matrix reinforced with discontinuous cellulose I. They suggest, the low solubility of the products and the decrease in the crystal size of cellulose I suggest that they are on the nanometer scale.

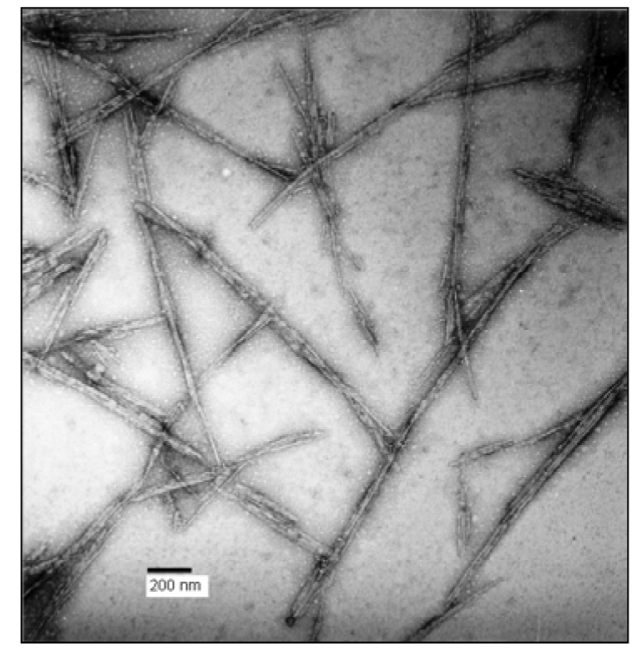

Figure $\mathbf{N}^{\circ} 17$ : TEM of nanocrystalline cellulose whiskers, obtained by acid hydrolysis of bacterial cellulose (Chatterjee, 2004).

In another area, Guo, et. al (2004) investigated the effects of nano-particles on cell morphology and foam expansion in the extrusion foaming of $\mathrm{mPE} /$ wood-fiber/nano-composites with a chemical blowing agent. The results indicate that the addition of clay generally decreases cell size, increases cell density and facilitates foam expansion. Furthermore, the foam material with added clay shows good char formation when it is burned. Adding nano-particles, to polymer wood composites, increased foam expansion. As expected, the SEM results demonstrated that cell morphology generally improved with respect to the cell size when clay was introduced in plastics. The DSC results showed that the crystallinity of the $\mathrm{mPE} /$ woodfiber/nano composites varied significantly with the woodfiber content and the clay content. The solubility was well correlated to the crystallinity. The addition of clay did not change the diffusivity of $\mathrm{CO}_{2}$ in the composites much. Finally, the foam material with clay showed good char formation when it was burned.

\section{BIOPOLYMERS FOR NANOCOMPOSITES}

A preliminary study using a biopolymer, polylactic acid (PLA), and two fillers with "nano" size (clays and calcium carbonate), was developed by Gacitua and Zhang (2005). An important effort is now conducted to improve general properties for PLA, which has a tremendous future as a polymer or reinforced polymer for automotive and other durable applications in a just few years.

Using only $2.5 \%$ of nanoclays or nanocalcium particles, they found a significant improvement in physical and mechanical properties for these nanocomposites. In these formulations, a biodegradable copolyester (Ecoflex) was used, 10\% weight based. 
Nanocomposites with nanoclays are $10 \%$ and $12 \%$ higher in terms of tensile and flexural strength, respectively, than nanocalcium based composites. Using TEM, morphological study for these nanocomposites showed that in general, both nanoparticles were well distributed in the PLA matrix. Calcium carbonate nanoparticles tend to be located inside of the Ecoflex domain and nanoclays tend to be aligned in the extrudate following the extrusion flow (see figure $\mathrm{N}^{\circ} 18$ ). At this point, information from these previous experiments do not allow to establish a supported discussion about this morphological effect. For the nanoclay composite, intercalated and exfoliated nanoparticles were observed. This effect increases the interaction, physical and mechanical, at the interface between filler and matrix, which helps to dissipate stresses and increase mechanical properties for the final nanocomposite.

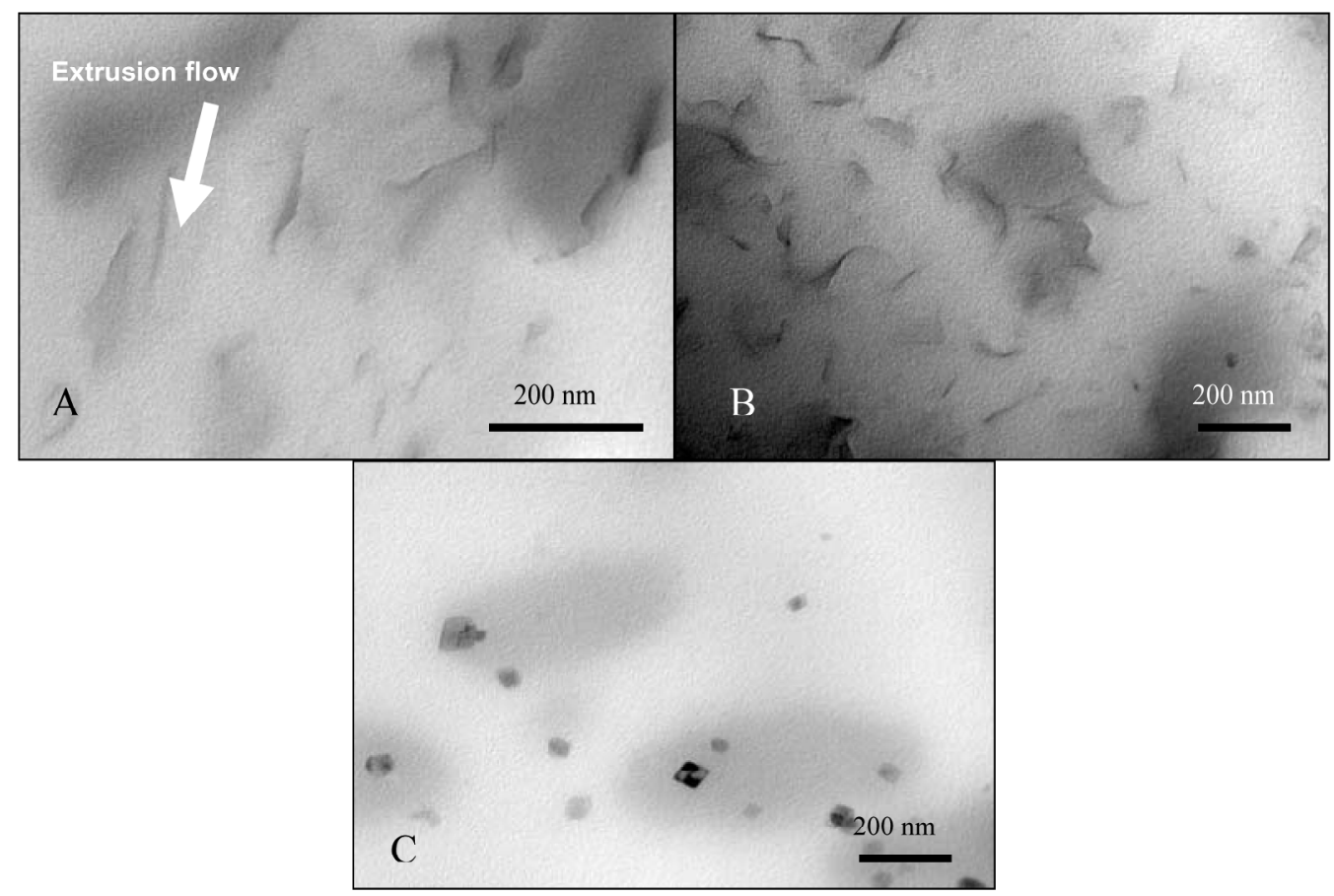

Figure $\mathbf{N}^{\circ}$ 18: a) Perfect alignment of nanoclays in a PLA matrix.

b) Intercalation and exfoliation of nanoclays in a PLA matrix.

c) Calcium carbonate nanoparticles in the Ecoflex domain (Gacitua and Zhang, 2005).

\section{CONCLUSIONS}

Significant research is needed to figure out the behavior of nano-interfaces, and this field can still be considered to be in its beginnings. In particular, the development of accurate nanomechanical models, and understanding of the properties of the polymer at the interface are required to address the outstanding issues of the polymer-nanoparticle interface and thus optimize the mechanical performance of polymer nanocomposites. It is believed that one of the main issues in preparing good polymer matrix nanocomposite samples is the good dispersion of the nanoparticles in a polymer matrix. Nanoparticles obtained from wood cell offer a great potential to make nanocomposites with biodegradable characteristics. 


\section{REFERENCES}

Ash, B. J.; Eitan, A.; Schadler, L. S. 2004. Polymer Nanocomposites with Particle and Carbon Nanotube Fillers. Dekker Encyclopedia of Nanoscience and nanotechnology.

Biswas, M.; Sinha, R. S. 2001.Recent progress in synthesis and evaluation of polymermontmorillonite nanocomposites. Adv Polym Sci.155:167-221.

Breuer, O.; Sundararaj, U. 2004. Big Returns From Small Fibers: A Review of Polymer/Carbon Nanotube Composites. Polymer Composites, 25(6): 630-645.

Chan, C.M.; Wu, J.; Li, J.; Cheung, Y. 2002. Polypropylene/calcium carbonate nanocomposite. Polymer 43, 2981-2992.

Chatterjee, A. P. 2004. Equation Methods in Macromolecular Science: Selected Applications. College of Environmental Science \& ForestryIntegral Department of Chemistry State University of New York.

Denault, J.; Labrecque, B., 2004. Technology Group on Polymer Nanocomposites - PNC-Tech. Industrial Materials Institute. National Research Council Canada, 75 de Mortagne Blvd. Boucherville, Québec, J4B 6Y4.

Ellis, T.; D'Angelo, J. 2003. Thermal and Mechanical Properties of a Polypropylene. Nanocomposite. Journal of Applied Polymer Science, 90: 1639-1647.

Fischer, H. 2003. Polymer nanocomposites: from fundamental research to specific applications. Materials Science and Engineering C 23: 763-772.

Gacitua, W. ; Zhang J. 2005. A preliminary study of mechanical, thermal and morphological properties for a bionanocomposite. WMEL, Washington State University, Pullman WA, USA.

Greene, M.; Kinser, C.; Kramer, D.; Pingree, L.; Hersam, M. 2004. Application of Scanning Probe Microscopy to the Characterization and Fabrication of Hybrid Nanomaterials. Microscopy Research and Technique 64:415-434.

Grunert, M.; Winter, W. 2002 Nanocomposites of Cellulose Acetate Butyrate Reinforced with Cellulose Nanocrystals. Journal of Polymers and the Environment, 10(1 and 2): 27-30.

Guo, G.; Wang, K.H.; Park, C.B.; Kim, Y.S.; Li G. 2004. Manufacture of Nano/mPE/WoodFiber Composite Foams in Extrusion. CSME 2004 Forum.

Gusev, A.A., 2001. Macromolecules 343081.

Helbert, W. ; Cavaille, J. Y. ; Dufresne, A. 1996.'Thermoplastic Nanocomposites Filled With Wheat Straw Cellulose Whiskers. Part I: Processing and Mechanical Behavior. Polymer composites, 17(4): 604-611.

Jordan, J.; Jacobb, K.; Tannenbaumc, R.; Sharafb, M.; Jasiukd, I. 2005. Experimental trends in polymer nanocomposites. Materials Science and Engineering A 393:1-11.

Ku, B.C.; Blumstein, A.; Kumar, J.; Samuelson, L. 2004.

Barrier Properties of Ordered Multilayer Polymer Nanocomposites. Dekker Encyclopedia of Nanoscience and nanotechnology. 
Mailhota, B.; Morlata, S.; Gardettea, J.L.; Boucardb, S.; Duchet, J.; Gerard, J.F. 2003. Photodegradation of polypropylene nanocomposites. Polymer Degradation and Stability 82:163-167.

Matsumura, H.; Sugiyama, J.; Glasser, W. 2000. Cellulosic Nanocomposites. I. Thermally Deformable Cellulose Hexanoates from Heterogeneous Reaction. Journal of Applied Polymer Science, 78: 2242-2253.

Mishra, J.; Hwang, K.J.; Ha, C.S. 2005. Preparation, mechanical and rheological properties of a thermoplastic polyolefin (TPO)/organoclay nanocomposite with reference to the effect of maleic anhydride modified polypropylene as a compatibilizer. Polymer 46 : 1995-2002.

Palaniyandi, V. ; Simonsen, J. 2004. Nanocrystalline Cellulose-filled High Density Polyethylene. Oregon State University Corvallis, OR.

Ray, S.S.; Okamoto, M. 2003 Polymer/layered silicate nanocomposites: a review from preparation to processing. Prog. Polym. Sci. 28: 1539-1641.

Sharma, P.; Miao, W.; Giri, A. 2004. Raghunathan S. Nanomaterials: Manufacturing, Processing, and Applications. Dekker Encyclopedia of Nanoscience and Nanotechnology. 2435 DOI: 10.1081/EENN 120009106.'Copyright by Marcel Dekker.

Schmidt, H. 2001. Nanoparticles by chemical synthesis, processing to materials an innovative applications. Applied organometallic chemistry, 15: 331-343.

Shelley, J.S.; Mather, P.T.; DeVries, K.L. 2001. Polymer 42: 5849-5858.

Silberglitt, R. 2004. Nanomaterials: New Trends. Dekker Encyclopedia of Nanoscience and nanotechnology.

Tidjani, A. 2005. Polypropylene-graft-maleic anhydrideenanocomposites: II e fire behaviour of nanocomposites produced under nitrogen and in air. Polymer Degradation and Stability 87: 43-49.

Van Es, M. 2001. Polymer-clay nanocomposites - the importance of particle dimensions, $\mathrm{PhD}$ Thesis, TU Delft,

Wagner, D.; Vaia, R. 2004. Nanocomposites: issues at the interface. Materials Today, November, 2004. ISSN:1369 7021 C Elsevier Ltd.

Winter, W.T. 2004. Ecocomposites Reinforced with Cellulose Nanoparticles: An Alternative to Existing Petroleum Based Polymer Composites. EPA Grant Number: R830897 1/15/2004-1/14/2007. Cellulose Res. Inst., SUNY-ESF, Syracuse, NY, 13210.

Wypych, F.; Satyanarayana, K. 2005. Functionalization of single layers and nanofibers: a new strategy to produce polymer nanocomposites with optimized properties. Journal of Colloid and Interface Science. In press. 